\title{
Efficacy of intensity-modulated radiotherapy with concurrent carboplatin in nasopharyngeal carcinoma
}

\author{
Anussara Songthong, Chakkapong Chakkabat, Danita Kannarunimit, \\ Chawalit Lertbutsayanukul ${ }^{1}$
}

Division of Radiation Oncology, Department of Radiology, Faculty of Medicine, Chulalongkorn University, King Chulalongkorn Memorial Hospital, Pathumwan, Bangkok, Thailand

Radiol Oncol 2015; 49(2): 155-162.

Received 30 June 2014

Accepted 1 October 2014

Correspondence to: Anussara Songthong, M.D., Division of Radiation Oncology, Department of Radiology, King Chulalongkorn Memorial Hospital, 1873 Rama IV Rd. Pathumwan, Bangkok 10330, Thailand. Phone: +662 256 4334; Fax: +662 256 4590; E-mail: anussara_pr@yahoo.com

Disclosure: No potential conflicts of interest were disclosed.

Background. The aim of the prospective phase II study was to evaluate the efficacy and toxicities of concurrent carboplatin with intensity-modulated radiotherapy (IMRT) in the treatment of nasopharyngeal carcinoma (NPC).

Patients and methods. Between October 2005 and November 2011, 73 stage II-IVB NPC patients received IMRT 70 Gy concurrently with three cycles of carboplatin (AUC 5) every three weeks, followed by three cycles of adjuvant carboplatin (AUC 5) and 5-FU (1,000 mg/m²/day for four days) every four weeks. All patients were evaluated for tumour response using response evaluation criteria in solid tumour (RECIST) criteria, survival analysis using Kaplan-Meier methods, and toxicities according to common terminology criteria for adverse events (CTCAE) version 4.0.

Results. At three months after chemoradiation, $82.2 \%$ and $17.8 \%$ of patients achieved complete and partial response, respectively. With a median follow-up of 48.1 months (1.3-97.8 months), $9.6 \%$ and $17.8 \%$ had local recurrence and distant metastasis, respectively. The median survival was not reached. A three-year overall survival was $83.6 \%$ and a progression-free survival was $65.3 \%$. Regarding treatment compliance, $97.2 \%, 68.5 \%$ and $69.8 \%$ completed radiation treatment, concurrent carboplatin and adjuvant chemotherapy, respectively. Grade 3-4 acute toxicities were oral mucositis (16.4\%), dysphagia (16.4\%), xerostomia (15.1\%) and haematotoxicity (6.8\%).

Conclusions. Carboplatin concurrently with IMRT provided excellent tumour response, manageable toxicities and good compliance. This should be considered as an alternative treatment for NPC patients.

Key words: intensity-modulated radiotherapy (IMRT); carboplatin; nasopharyngeal carcinoma

\section{Introduction}

Nasopharyngeal carcinoma (NPC) is one of the most common head and neck neoplasms among Asian people. The overall incidence of NPC in Southeast Asia is 6.5 and 2.6 per 100,000 personyears in males and females, respectively. ${ }^{1}$ In Thailand, the age-standardized incidence rates of NPC are approximately 3.7 and 1.2 per 100,000 in males and females, respectively. ${ }^{2}$

Meta-analysis showed that chemotherapy plays an important role in the treatment of this disease. ${ }^{3}$ Al Saraff et al. (Intergroup 0099) demonstrated sig- nificant benefits of additional cisplatin in terms of both disease free survival and overall survival, when used concurrently with radiation followed by a combination of cisplatin and 5-fluorouracil chemotherapy for three cycles. ${ }^{4}$ Thus, this regimen has become standard of care for nasopharyngeal carcinoma despite the low compliance rate (55-63\%) in this trial. Significant side effects of cisplatin include nausea and vomiting, renal, neurological and ototoxicity. Additionally, during highdose cisplatin administration, adequate hydration and volume monitoring are needed and require hospital admission. Recently, Chan et al. proposed 
a low-dose weekly cisplatin that could be administered in an outpatient setting and provides good patient compliance. ${ }^{5}$

Based on similar radiosensitizing properties of carboplatin and cisplatin along with pre-clinical data that demonstrated an enhanced radiation effect from concurrent carboplatin in tumour cells, some physicians use carboplatin as an alternative regimen to avoid serious cisplatin toxicities, especially renal, gastrointestinal and neurotoxicity. ${ }^{6-10}$

Many studies have shown comparable response rates and survival outcomes with acceptable toxicities and better compliance from carboplatin. ${ }^{11-14}$ However, the radiation technique in those studies was the conventional technique. More recently, intensity-modulated radiotherapy (IMRT) has been proven in NPC treatment for its efficacy and its advantages over conventional techniques and has been considered as a standard radiation technique for NPC. ${ }^{15-17}$

The objectives of this study are to evaluate efficacy and toxicities using IMRT concurrently with carboplatin, followed by adjuvant carboplatin and 5-fluorouracil (5-FU) chemotherapy for the treatment of NPC.

\section{Patients and methods}

\section{Patients and methods}

Between October 2005 and November 2011, newly diagnosed NPC patients were accrued for this prospective phase II study after obtaining the institutional review board approval (RA 13/49). The eligibility criteria included those aged 18 years old and above; histologically confirmed non-metastatic nasopharyngeal carcinoma stage II-IVB according to the $7^{\text {th }}$ edition of the American Joint Committee on Cancer Staging System (AJCC 2010); Eastern Cooperative Oncology Group (ECOG) performance status 0-2; adequate hematologic and renal function, defined by with blood cells (WBC) $\geq 4,000 /$ $\mathrm{mL}$, platelet count $\geq 100,000 / \mathrm{mL}$, serum creatinine $\leq 1.5 \mathrm{mg} / \mathrm{dL}$ or calculated creatinine clearance $\geq 60$ $\mathrm{ml} / \mathrm{min}$. Patients with distant metastasis; previous radiation and/or chemotherapy treatment less than six months prior to the study entry; other malignancy except non-melanoma skin cancer or a carcinoma of non-head and neck origin, controlled for at least five years; active infection; major medical or psychiatric condition or pregnancy were excluded.

All eligible patients received a pre-treatment evaluation including complete history and physical examination, endoscopic biopsy, routine labo- ratory tests for hematologic, renal and hepatic function as well as a dental and nutritional evaluation before the treatment. Radiological investigations consisted of computed tomography (CT) scan or magnetic resonance imaging (MRI) of the nasopharynx, chest radiography, ultrasound of upper abdomen and bone scintigraphy. Positron emission tomography (PET) scan was optional. A pathologic confirmation of NPC was performed and re-classified according to WHO subtype. ${ }^{18}$

\section{Treatment protocol}

Each patient underwent contrast-enhanced CT simulation with a long thermoplastic mask. The GTVs and CTVs were contoured according to RTOG guidelines. There were two planning target volumes (PTVs): PTV-high risk (PTV-HR), defined as primary tumour and gross lymphadenopathy with appropriate margin, and PTV-low risk (PTVLR), defined as PTV-HR plus elective lymph node region. The prescription dose was $50 \mathrm{~Gy}$ in 25 fractions to PTV-LR followed by a boost of $20 \mathrm{~Gy}$ in 10 fractions, called sequential IMRT (SEQ). Recently, a simultaneous integrated boost (SIB) technique was developed and applied in last few patients with total dose of $70 \mathrm{~Gy}$ and $56 \mathrm{~Gy}$ in 33 fractions for PTV-HR and PTV-LR, respectively. Normal tissue constraints were used according to our institutional protocol (adopted from RTOG 0225 and 0615 study protocols) and are described in Table 1.

All patients received IMRT concurrently with three cycles of carboplatin (AUC 5) every three weeks, followed by three cycles of adjuvant carboplatin (AUC 5) and 5-FU (1,000 mg/m² /day for four days) every four weeks.

During the concurrent and the adjuvant treatment, patients were assessed weekly. Dose modification and proper management were performed according to patients' toxicity grading. The response of the primary tumour and lymph node was evaluated at three months after the last fraction of radiotherapy by endoscopic examination and $\mathrm{CT}$ scan. Other imaging was performed if indicated.

\section{Statistical analysis}

Data collection consisted of patient characteristics including age, sex and ECOG performance status; disease characteristics including pathologic WHO subtype and TNM staging; and treatment modalities including radiation treatment technique, radiation dose, duration of radiation treatment as well as compliance with radiation and chemotherapy. 
TABLE 1. Dose volume constraints of normal tissue

\begin{tabular}{|c|c|c|c|}
\hline \multirow{2}{*}{ Organ at risk } & \multirow{2}{*}{$\begin{array}{l}\text { Maximum } \\
\text { dose (Gy) }\end{array}$} & \multicolumn{2}{|c|}{$\begin{array}{l}\text { Dose volume } \\
\text { constraints }\end{array}$} \\
\hline & & $\begin{array}{l}\text { Dose } \\
\text { (Gy) }\end{array}$ & $\begin{array}{c}\text { Maximum } \\
\text { volume }\end{array}$ \\
\hline Spinal cord & 50 & 45 & $1 \mathrm{cc}$ \\
\hline Brain stem & 60 & 54 & $1 \mathrm{cc}$ \\
\hline One parotid gland & & 26 & $50 \%$ \\
\hline Optic nerve & 54 & & \\
\hline Cochlear & & 46 & $50 \%$ \\
\hline Eyes & 24 & & $50 \%$ \\
\hline Lens & 6 & & \\
\hline Mandible & 70 & 53 & $50 \%$ \\
\hline Oral cavity & 60 & 40 & $50 \%$ \\
\hline Vocal cord & 58 & 45 & $50 \%$ \\
\hline
\end{tabular}

Maximum dose (Dmax) defined as radiation dose encompasses $1 \%$ of each organ-at-risk volume

All patients were evaluated for tumour response using response evaluation criteria in solid tumour (RECIST) criteria, survival outcomes using Kaplan-Meier methods, and acute and late toxicities according to common terminology criteria for adverse events (CTCAE) version 4.0.

Primary endpoints were progression-free survival (PFS) and overall survival (OS). PFS was defined as the time period since the initial treatment of NPC until disease recurrence or progression or death. OS was defined as the time period between the initial treatment of NPC and any cause of death. Survival analyses were computed using the Kaplan-Meier method and log-rank test. P-value of 0.05 or less was applied to define significance. Statistical Packages for Social Sciences (SPSS) software version 17.0 was used for the statistical analysis. Secondary endpoints were disease control and treatment-related toxicities.

The sample size calculation was based on the proportion of expected death (mortality rate) with $95 \%$ confidence interval. We employed $18.1 \%$ mortality rate for concurrent radiation with carboplatin according to the results of a randomized study of Chitapanarux et al. ${ }^{13}$ Allowing 20\% dropout p-value of $0.05,69$ participants were planned to be enrolled in the study.

\section{Results}

\section{Patient and disease characteristics}

A total of 73 patients diagnosed with NPC and treated between October 2005 and November
TABLE 2. Patients and disease characteristics

\begin{tabular}{|c|c|c|}
\hline & $N(73)$ & $\%$ \\
\hline $\begin{array}{l}\text { Age, years } \\
\text { Mean (range) }\end{array}$ & $54.4(24-76)$ & \\
\hline $\begin{array}{l}\text { Sex } \\
\text { Male } \\
\text { Female }\end{array}$ & $\begin{array}{l}49 \\
24\end{array}$ & $\begin{array}{l}67.1 \% \\
32.9 \%\end{array}$ \\
\hline $\begin{array}{l}\text { Performance status } \\
\text { ECOG } 0 \\
\text { ECOG } 1\end{array}$ & $\begin{array}{l}67 \\
6\end{array}$ & $\begin{array}{l}91.8 \% \\
8.2 \%\end{array}$ \\
\hline $\begin{array}{l}\text { WHO classification } \\
\text { Type II (Non-keratinizing SCCA) }\end{array}$ & 73 & $100.0 \%$ \\
\hline $\begin{array}{c}\text { T stage } \\
1 \\
2 \\
3 \\
4\end{array}$ & $\begin{array}{l}15 \\
26 \\
23 \\
9\end{array}$ & $\begin{array}{l}20.6 \% \\
35.6 \% \\
31.5 \% \\
12.3 \%\end{array}$ \\
\hline $\begin{array}{c}\text { N stage } \\
0 \\
1 \\
2 \\
3 a \\
3 b\end{array}$ & $\begin{array}{l}5 \\
19 \\
41 \\
5 \\
3\end{array}$ & $\begin{array}{l}6.8 \% \\
26.0 \% \\
56.2 \% \\
6.9 \% \\
4.1 \%\end{array}$ \\
\hline $\begin{array}{l}\text { M stage } \\
0\end{array}$ & 73 & $100.0 \%$ \\
\hline $\begin{array}{l}\text { Stage grouping } \\
\text { II } \\
\text { III } \\
\text { IV A } \\
\text { IV B }\end{array}$ & $\begin{array}{l}16 \\
42 \\
12 \\
3\end{array}$ & $\begin{array}{l}22.0 \% \\
57.5 \% \\
16.4 \% \\
4.1 \%\end{array}$ \\
\hline
\end{tabular}

SCCA $=$ Squamous cell carcinoma

2011 were accrued. Patient and disease characteristics are listed in Table 2. The mean age was 54.4 years (range 24-76 years). The majority of patients $(67.1 \%)$ were males. All were in good performance status and had non-serious comorbidities. The histological subtype, according to WHO classification, was non-keratinizing squamous cell carcinoma (NK-SCCA) in every patient, which could be further identified as undifferentiated NK-SCCA in most patients $(83.6 \%)$. Approximately half of patients had stage III disease.

\section{Radiation treatment}

Whole-neck IMRT was planned using the Eclipse treatment planning system. The majority of patients $(91.8 \%)$ was treated with the SEQ-IMRT technique. The rest were treated with the SIBIMRT technique. Seventy-two patients (98.6\%) completed a course of radiation. One patient could not complete the course of radiation and the treatment interruption of 38 days occurred in another patient; both resulted from intolerable toxicity. The average PTV-HR and PTV-LR dose were 69.95 Gy (range 58-76 Gy) and 50.82 Gy (range 42-62 Gy), respectively. The median duration of the radiation treatment was 55 days (range 14-93 days). 


\section{Chemotherapy}

Seventy-two patients $(98.6 \%)$ received concurrent carboplatin and radiation; 50 patients $(68.5 \%)$ received all three cycles of chemotherapy as planned. The compliance of chemotherapy treatment is detailed in Table 3.

\section{Clinical outcome}

The median follow-up time was 48.1 months (6.1-97.8 months). At three months after completion of radiotherapy, a complete response (CR) was achieved in 60 patients $(82.2 \%)$ while 13 patients (17.8\%) achieved a partial response (PR). Regarding the site of the tumour response, $94.5 \%$ of patients achieved CR at the primary (nasopharyngeal) site while $83.6 \%$ achieved CR at regional lymph node

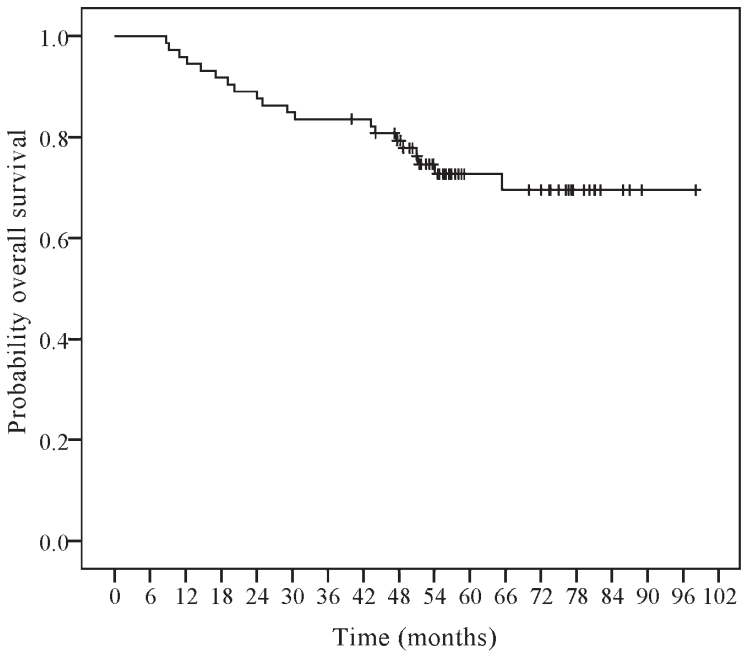

(B)

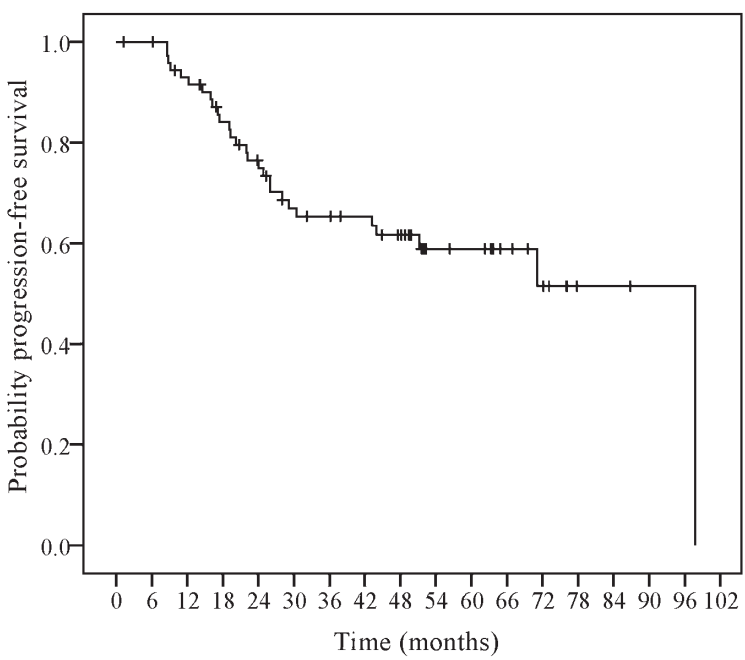

FIGURE 1. Overall and progression-free survival of patients with nasopharyngeal carcinoma treated with intensity-modulated radiotherapy and concurrent carboplatin.
TABLE 3. Compliance of chemotherapy treatment

\begin{tabular}{lcc}
\hline \multirow{2}{*}{$\begin{array}{l}\text { Number } \\
\text { of cycles }\end{array}$} & $\begin{array}{c}\text { N (\%) } \\
\text { Concurrent } \\
\text { carboplatin }\end{array}$ & $\begin{array}{c}\text { Adjuvant } \\
\text { carboplatin/5-Fu }\end{array}$ \\
\cline { 2 - 3 } 0 & $1(1.4 \%)$ & $4(5.5 \%)$ \\
1 & $2(2.7 \%)$ & $8(11 \%)$ \\
2 & $20(27.4 \%)$ & $10(13.7 \%)$ \\
3 & $50(68.5 \%)$ & $51(69.8 \%)$ \\
Total & $73(100 \%)$ & $73(100 \%)$ \\
\hline
\end{tabular}

sites. Patients who achieved PR received a further treatment: a radiotherapy boost of $30 \mathrm{~Gy}$ in 10 fractions to any residual disease at the primary site. Patients with small residual lymph node(s) had a radiation boost of $15 \mathrm{~Gy}$ in 5 fractions while those with a larger residual disease in the neck underwent a salvage neck dissection. No additional systemic therapy was given after the patients completed three chemotherapy cycles.

During the follow-up period, seven patients $(9.6 \%)$ and 13 patients $(17.8 \%)$ experienced local and distant failure, respectively. None of the patients had regional recurrence or both, local/regional and distant failure. The median time to local and distant recurrence was 20.3 months and 22.2 months, respectively. The most common sites of metastasis were bone $(8.2 \%)$, liver $(6.8 \%)$ and lung $(4.1 \%)$.

At the last follow-up, 45 patients $(61.6 \%)$ were alive without disease while eight patients $(10.9 \%)$ had disease recurrence. There were 20 deaths $(27.4 \%) ; 14$ patients died from the progression of the disease.

\section{Survival outcome}

Median OS was not reached. Median PFS was 71 months. Three-year OS and PFS were $83.6 \%$ and $65.3 \%$, respectively and at 5 years they were $72.7 \%$ and $58.9 \%$, respectively, as shown in Figure 1.

\section{Toxicities}

The toxicities were classified as acute and late toxicities using a 90-day cut-off point after the completion of chemoradiation. Acute toxicity consisted of symptoms developing during the concurrent and the adjuvant treatment. During concurrent chemoradiation, all patients experienced some degrees of acute toxicities, most of which were mild (grade $1-2)$. The most common grade 3-4 toxicities were 
mucositis $(16.4 \%)$, dysphagia $(16.4 \%)$ and xerostomia $(15.1 \%)$. Only two patients $(2.7 \%)$ had severe nausea and vomiting. Twelve patients (16.4\%) needed nasogastric tube insertion. Weight loss of more than $20 \%$ (grade 3 ) occurred in 5 patients $(6.8 \%)$ during concurrent chemoradiation and in 24 patients $(32.9 \%)$ during the adjuvant period. During adjuvant chemotherapy, most patients recovered from mucositis, xerostomia and dysphagia. Grade 3 or more hematologic toxicities developed in 6 patients $(8.2 \%)$. Three $(4.1 \%)$ and two patients $(2.7 \%)$ developed grade $3-4$ neutropenia and thrombocytopenia, respectively. A renal function impairment was not found. No patient developed grade 5 toxicity during the concurrent and the adjuvant treatment. The incidence of acute toxicities is described in Table 4.

Eighteen patients $(28.6 \%)$ had grade 3 weight loss at one year after chemoradiation. Most patients regained their weight within two years. Grade 2 xerostomia was found in 10 patients $(13.7 \%)$ and three patients (4.1\%) at 6-month and 12-month follow-up, while no patient had grade 2 xerostomia at the 24-month point. None of the patients experienced grade 3-4 gastrointestinal and dermatologic toxicities during follow-up. There was no renal toxicity among these patients.

\section{Discussion}

Nasopharyngeal carcinoma is one of the most common head and neck cancer in Southeast Asia and has a different natural history and prognosis from other cancers in this region. The current standard treatment of locally advanced NPC is concurrent chemoradiation followed by adjuvant chemotherapy. ${ }^{3-5}$ According to a meta-analysis from eight trials involving 1,753 patients, chemotherapy resulted in an absolute survival benefit of $6 \%$ (from $56 \%$ to $62 \%$ ) and an event-free survival benefit of $10 \%$ (from $42 \%$ to $52 \%$ ) at five years. This study also concluded that the concurrent trials showed significant survival benefit with hazard ratio (HR) of 0.60 (95\% CI, 0.48-0.76). ${ }^{3}$ Another meta-analysis from 10 randomized clinical studies with a total of 2,450 patients supported that concurrent chemoradiation improved survival by $20 \%$ at five years with a pooled HR of 0.48 (95\% CI, 0.32-0.72).${ }^{19}$ The landmark study by Al Saraff et al. (INT 0099) ${ }^{4}$ supported the standard treatment of nasopharyngeal carcinoma using concurrent radiation with cisplatin followed by cisplatin and 5FU with 5-year OS and DFS of $67 \%$ and $58 \%$, respectively. Although
TABLE 4. Acute toxicity during treatment

\begin{tabular}{lcccc}
\hline \multirow{2}{*}{ Toxicities } & \multicolumn{2}{c}{$\begin{array}{c}\text { Acute toxicity } \\
\text { During chemoradiation }\end{array}$} & $\begin{array}{c}\text { Acute toxicity } \\
\text { During adjuvant period }\end{array}$ \\
\cline { 2 - 5 } & $\mathbf{0 - 2}$ & $\mathbf{3 - 5}$ & $\mathbf{0 - 2}$ & $\mathbf{3 - 5}$ \\
\hline Constitutional symptoms & & & & \\
$\quad$ Fatigue & $72(98.6 \%)$ & $1(1.4 \%)$ & $71(97.3 \%)$ & $2(2.7 \%)$ \\
$\quad$ Anorexia & $71(97.3 \%)$ & $2(2.7 \%)$ & $73(100 \%)$ & 0 \\
Weight loss & $68(93.2 \%)$ & $5(6.8 \%)$ & $49(67.1 \%)$ & $24(32.9 \%)$ \\
Gastrointestinal & & & & \\
Oral mucositis & $61(83.6 \%)$ & $12(16.4 \%)$ & $73(100 \%)$ & 0 \\
Xerostomia & $62(84.9 \%)$ & $11(15.1 \%)$ & $73(100 \%)$ & 0 \\
Dysphagia & $61(83.6 \%)$ & $12(16.4 \%)$ & $73(100 \%)$ & 0 \\
Nausea & $71(97.3 \%)$ & $2(2.7 \%)$ & $73(100 \%)$ & 0 \\
Vomiting & $71(97.3 \%)$ & $2(2.7 \%)$ & $73(100 \%)$ & 0 \\
$\quad$ Diarrhea & $73(100 \%)$ & 0 & $73(100 \%)$ & 0 \\
Dermatitis & $73(100 \%)$ & 0 & $73(100 \%)$ & 0 \\
Hematologic & & & & \\
$\quad$ Anaemia & $73(100 \%)$ & 0 & $72(98.6 \%)$ & $1(1.4 \%)$ \\
Neutropenia & $68(93.2 \%)$ & $5(6.8 \%)$ & $70(95.9 \%)$ & $3(4.1 \%)$ \\
Thrombocytopenia & $72(98.6 \%)$ & $1(1.4 \%)$ & $71(97.3 \%)$ & $2(2.7 \%)$ \\
Creatinine & $73(100 \%)$ & 0 & $73(100 \%)$ & 0 \\
Total & $73(100 \%)$ & 0 & $73(100 \%)$ & 0 \\
\hline
\end{tabular}

cisplatin is a widely accepted regimen, its toxicity, including nausea, vomiting, ototoxicity, neurotoxicity and nephrotoxicity, may lead to poor compliance; only $63 \%$ and $55 \%$ of patients completed concurrent and adjuvant chemotherapy in INT 0099. ${ }^{4}$

Carboplatin has come into interest due to its lesser side effects, especially gastrointestinal and nephrogenic side effects. The advantages of carboplatin are tolerable toxicity leading to better compliance and its capability of out-patient administration, thus reducing hospitalization, cost of the treatment and workload of medical personnel. Eisenberger indicated that $100 \mathrm{mg} / \mathrm{m}^{2} /$ week of carboplatin was well tolerated when given concurrently with radiation in locally advanced head and neck cancers. ${ }^{20}$ Unfortunately for NPC, a prospective phase I/II study from Canada of concurrent carboplatin in 47 patients reported probably inferior OS and PFS with acceptable toxicity compared to INT 0099. With the median follow-up of 23.1 months, 3 -year OS and PFS were $56 \%$ and $58 \%$, respectively. ${ }^{11}$ Nevertheless, different WHO histological subtypes may result in different natural history of disease and response between Caucasian and Asian people. The randomized controlled trial from Thailand, comparing carboplatin-based chemotherapy with an INT 0099 regimen in 220 patients demonstrated a non-inferior survival outcome. Three-year OS and DFS in the carboplatin arm were $79.2 \%$ and $60.9 \%$ compared with $77.7 \%$ and $63.4 \%$ in the cisplatin arm. They also showed better compliance to treatment in carboplatin arm, $73 \%$ versus $59 \% .{ }^{13}$ Another report from Thailand 
TABLE 5. Comparison of treatment schedule, compliance and outcome between studies on concurrent chemoradiation with carboplatin in NPC patients and INT 0099 trial; and RTOG 0225 using IMRT technique

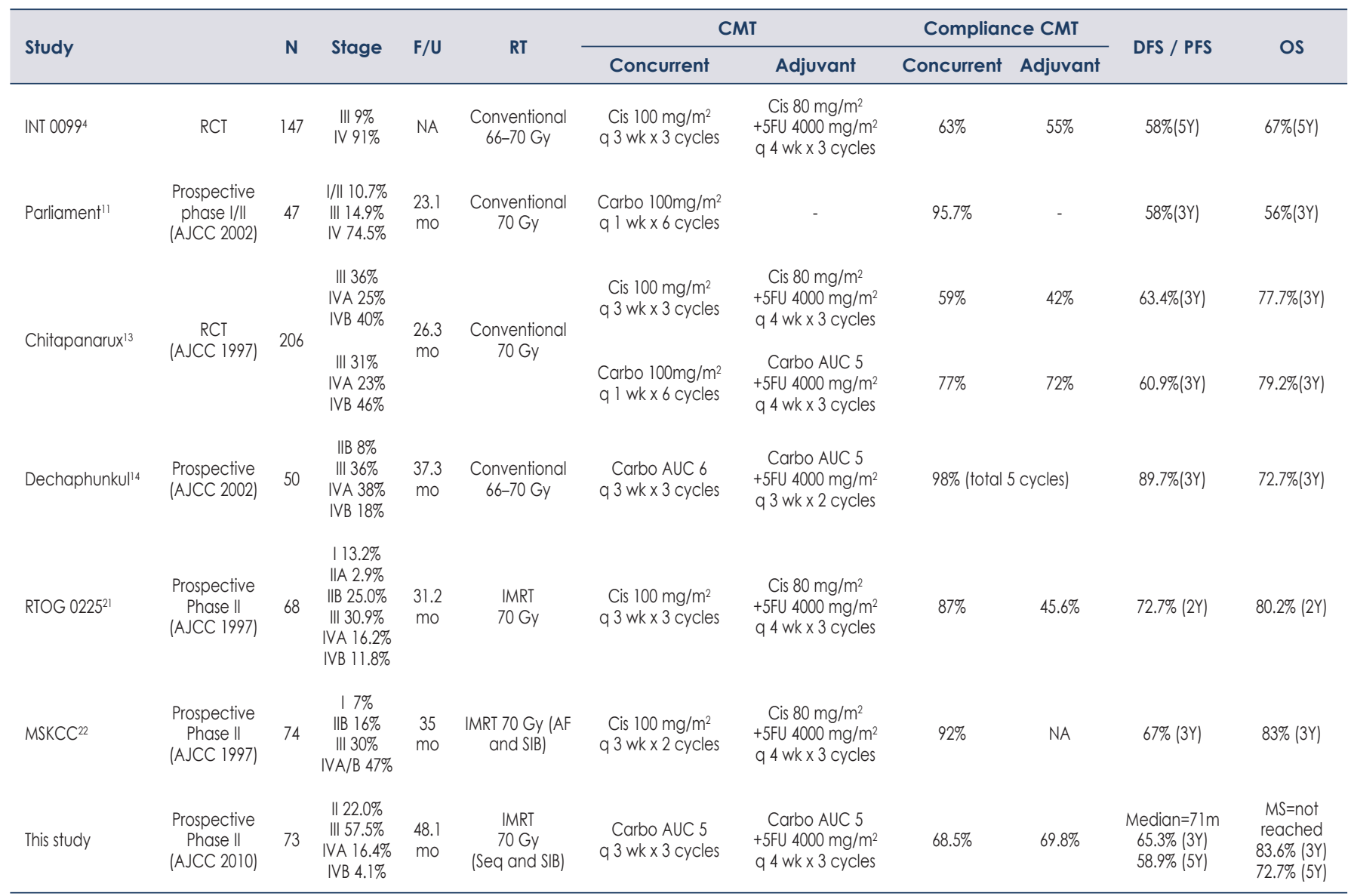

$\mathrm{AF}=\mathrm{Accelerated}$ fractionation (here, hyperfractionated concomitant boost) AJCC $=$ American Joint Committee on Cancer Staging; Carbo $=\mathrm{Carboplatin} ;$ Cis $=$ Cisplatin $\mathrm{CMT}=$ Chemotherapy; DFS = Disease-free survival; F/U = Follow-up time; $5 \mathrm{FU}=5$-fluorouracil; $\mathrm{mo}=$ months; NA = Not available; OS = Overall survival; PFS = Progression-free survival; $\mathrm{RCT}$ = randomized-controlled trial; RT = Radiation treatment; SIB = simultaneous integrated boost; wk = week

that included 50 patients using concurrent chemoradiation with carboplatin followed by carboplatin and 5FU in NPC showed good results and tolerability. The 3-year OS and PFS were $72.7 \%$ and $89.7 \%$, respectively. ${ }^{14}$

Although the efficacy of concurrent carboplatin with radiation in NPC was demonstrated, these studies used a conventional radiation technique ${ }^{11,13,14}$ whereas in current practice, the standard radiation technique for NPC is IMRT, which has demonstrated significantly better salivary flow rate and quality of life in NPC patients compared with conventional techniques. ${ }^{15-17}$ Additionally, the recent RTOG phase II trial 0225 and a prospective study from Memorial Sloan Kettering Cancer Center (MSKCC) reported promising results of tumour control and toxicities by using IMRT and concurrent cisplatin. ${ }^{21,22}$ In RTOG 0225 with 68 patients, 2-year OS and PFS were $80.2 \%$ and
$72.7 \%$, respectively. In the MSKCC study with 74 patients, 3-year OS and PFS were $83 \%$ and $67 \%$, respectively. In this study, using concurrent carboplatin with IMRT, our 3-year OS and PFS were $83.6 \%$ and $65.3 \%$, which were comparable with those previous studies. Moreover, approximately $70 \%$ of patients completed three cycles of adjuvant chemotherapy.

Distant metastasis is the major reason of failure in nasopharyngeal carcinoma. The rate of distant metastasis reported in many series was $14.7 \%-22 \%$ in cisplatin-based chemotherapy series compared with $14.3 \%-29.8 \%$ in carboplatin-based series. $411-14,18,21,22$ In our study, the crude distant metastasis rate was $17.8 \%$, which was comparable with the results from studies using either of the two chemotherapy regimens. The most common sites of metastasis were bone $(8.2 \%)$, liver $(6.8 \%)$ and lung (4.1\%), giving the 3- and 5-year distant 
metastasis-free survival (DMFS) of $82 \%$ and $77.1 \%$, respectively.

With regards to toxicity, because different criteria were used for evaluation, comparing the toxicity among several published trials including our study might not be appropriate. Compared with RTOG 0225, which used similar toxicity evaluation criteria, Common Terminology Criteria for Adverse Events (CTCAE), overall acute grade 3 toxicity was $61.8 \%{ }^{21}$, while it was $24.7 \%$ in our study. Acute grade 3 mucositis, defined as confluent pseudomembranous reaction (contiguous patches generally $>1.5 \mathrm{~cm}$ in diameter) in CTCAE version 2.0 and as severe pain or interference with oral intake in CTCAE version 4.03 , was $29.4 \%$ in the RTOG study compared to $16.4 \%$ in our study. There was no patient who experienced acute grade 5 mucositis in this trial but one patient $(1.3 \%)$ did in the RTOG study. According to Dechapunkul, using conventional radiation and RTOG acute toxicity criteria in which grade 3 mucositis was defined as confluent fibrinous mucositis or severe pain requiring narcotics, grade 3-4 mucositis was reported in $42 \%$ of total 50 patients. ${ }^{14}$ In contrast, in the Chitapanarux study, using conventional radiation technique and RTOG acute toxicity criteria, the rate of mucous membrane toxicity was very low: $0 \%$ in the cisplatin arm and 5\% in the carboplatin arm. However, the rate of nasogastric tube insertion was as high as $48 \%$ and $22 \%$ in the cisplatin and carboplatin arm, respectively, compared with $16.4 \%$ in our study.

Grade 3 or higher nausea and vomiting rates during chemoradiation in our study were comparable to those of carboplatin studies and was less than cisplatin studies, for example, $2.7 \%$ in our study, $0 \%$ in Chitapanarux study, $8 \%$ in Dechapunkul study versus $19.2 \%$ in INT 0099 study.

The rate of grade 3 dermatitis, which was defined as confluent moist desquamation $\geq 1.5 \mathrm{~cm}$ diameter and not confined to skin folds or pitting oedema in CTCAE version 2.0 and RTOG toxicity criteria and as moist desquamation other than skin folds and creases or bleeding induced by minor trauma or abrasion in CTCAE 4.03 , was $13.2 \%$ in the RTOG 0225 study, 3\% and 6\% in the cisplatin and carboplatin arm in the Chitapanarux study, and none in our study.

Late grade 2 xerostomia at 12 -month period in our study was $4.1 \%$ compared with $13.5 \%$ reported in RTOG 0225 and $24 \%$ in the Chitapanarux study.

The treatment schedule, compliance and outcome in each study are demonstrated in Table 5.
One of the major concerns of carboplatin administration is hematologic toxicity. In our study, $8.2 \%$ experienced grade $3-4$ hematologic toxicity, mainly neutropenia (6.8\%) and thrombocytopenia $(1.4 \%)$, which was comparable to the Chitapanarux study in which $10 \%$ and $2 \%$ of patients experienced grade 3 neutropenia and thrombocytopenia in the carboplatin arm.

This is the first study to our knowledge that demonstrated the efficacy and feasibility of carboplatin concurrently with IMRT in NPC and that this treatment can be applied as an alternative chemotherapy regimen, especially in vulnerable patients or those not suitable for standard cisplatin regimen.

\section{Conclusions}

Carboplatin concurrently with IMRT provided excellent tumour response, manageable toxicities and good compliance. This should be considered as alternative to standard treatment with cisplatin for NPC patients.

\section{References}

1. International Agency for Research on Cancer, World Health Organisation, International Association of Cancer Registries. Nasopharyngeal carcinoma. In: Curado MP, Edwards B, Shin HR, Storm H, Ferlay J, Heanue M, et al, editors. Cancer in five continents, vol IX. IARC Scientific Publication No. 160. Lyon: IARC; 2007.

2. Nasopharyngeal carcinoma. In: Khuhaprema T, Srivatanakul P, Attsara A, Sriplung $\mathrm{H}$, Wiangnon $\mathrm{H}$, Sumitsawan $\mathrm{Y}$, editors. Cancer in Thailand. Bangkok: Ministry of health; 2010. p. 16-17.

3. Baujet B, Audry H, Bourhis J, Chan AT, Onat H, Chua DT, et al. Chemotherapy in locally advanced nasopharyngeal carcinoma: an individual patient data meta-analysis of eight randomized trials and 1753 patients. Int J Radiat Oncol Biol Pys 2006; 64: 47-56.

4. Al-Sarraf M, LeBlanc M, Giri PG, Fu KK, Cooper J, Vuong T, et al. Chemoradiotherapy versus radiotherapy in patients with advanced nasopharygeal cancer: Phase III randomized Intergroup study 0099. J Clin Oncol 1998; 16: 1310-7.

5. Chan AT, Leung SF, Ngan RK, Teo PM, Lau WH, Kwan WH, et al. Overall survival after concurrent cisplatin-radiotherapy compared with radiotherapy alone in locoregionally advanced nasopharyngeal carcinoma. J Natl Cancer Inst 2005; 97: 536-9.

6. Douple EB, Richmond RC, O'Hara JA, Coughlin CT. Carboplatin as a potentiator of radiation therapy. Cancer Treat Rev 1985; 12(Suppl A): 111-24.

7. Coughlin CT, Richmond RC. Biologic and clinical developments of cisplatin combined with radiation: Concepts, utility, projections for new trials, and the emergence of carboplatin. Semin Oncol 1989; 16: 31-43.

8. Calais G1, Alfonsi M, Bardet E, Sire C, Germain T, Bergerot P, et al. Randomized trial of radiation therapy versus concomitant chemotherapy and radiation therapy for advanced-stage oropharynx carcinoma. I Natl Cancer Inst 1999; 91: 2081-6.

9. Calais $\mathrm{G}$, Le Floch $\mathrm{O}$. Concomitant radiotherapy and chemotherapy in the treatment of cancer of the upper respiratory and digestive tracts. Bull Cancer Radiother 1996; 83: 321-9. 
10. Muggia FM. Overview of carboplatin: Replacing complementing and extending the therapeutic horizons of cisplatin. Semin Oncol 1989; 16(Supp 5): $7-13$

11. Paliament M, Jha N, Rapp E, Smith C, MacKinnon J, Nabholtz JM, et al. Concurrent weekly carboplatin and radiotherapy for nasopharyngeal carcinoma: report of a joint phase II study. Radiother Oncol 2001; 58: 131-6.

12. Okita J, Hatta C, Terada T, Saeki N, Ogasawara H, Kakibuchi M, et al. Concurrent chemo-radiotherapy for nasopharyngeal carcinoma. Auris Nasus Larynx 2004; 31: 43-7.

13. Chitapanarux I, Lorvidhaya V Kamnerdsupaphon $P$, Sumitsawan $Y$ Tharavichitkul E, Sukthomya V, et al. Chemoradiation comparing cisplatin versus carboplatin in locally advanced nasopharyngeal cancer: Randomised, non-inferiority, open trial. Euro J of Cancer 2007; 43: 1399-406.

14. Dechaphunkul T, Pruegsanusak K, Sangthawan D, Sanpaeravong $P$. Concurrent chemoradiotherapy with carboplatin followed by carboplatin and 5- fluorouracil in locally advanced nasopharyngeal carcinoma. Head Neck Oncol 2011; 3: 30

15. Pow EH, Kwong DL, McMillan AS, Wong $M C$, Sham JS, Leung $L H$, et al Xerostomia and quality of life after intensity-modulated radiotherapy vs conventional radiotherapy for early-stage nasopharyngeal carcinoma: initial report on a randomized controlled clinical trial. Int Radiat Oncol Biol Phys 2006; 66: 981-91.

16. Kam MK, Leung SF, Zee B, Chau RM, Suen JJ, Mo F, et al. Prospective randomized study of intensity- modulated radiotherapy on salivary gland function in early-stage nasopharyngeal carcinoma patients. J Clin Oncol 2007; 25: 4873-9.

17. Peng G, Wang T, Yank KY, Zhang $S$, Zhang $T$, Li $Q$, et al. A prospective, randomized study comparing outcomes and toxicities of intensity-modulated radiotherapy vs. conventional two-dimensional radiotherapy for the treatment of nasopharyngeal carcinoma. Radiother Oncol 2012; 104: 286-93.

18. Chan J, Bray F, McCarron P, Foo W, Lee AWM, Yip T, et al. Nasopharyngeal carcinoma. In: Barnes EL, Eveson JW, Reichart P, Sidransky D, editors. Pathology and genetics of head and neck tumor. World Health Organization classification of tumours. Lyon: IARC Press; 2005. p. 85.

19. Langendijk JA, Leemans CR, Buter J, Berkhof J, Slotman BJ. The additional value of chemotherapy to radiotherapy in locally advanced nasopharyngeal carcinoma: a meta-analysis of the published literature. J Clin Oncol 2004; 22: $4604-12$.

20. Eisenberger $M$, Jacobs $M$. Simultaneous treatment with single-agent chemotherapy and radiation for locally advanced cancer of head and neck. Semin Oncol 1992; 4(Suppl11): 41-6.

21. Lee N1, Harris J, Garden AS, Straube W, Glisson B, Xia P, et al. Intensitymodulated radiation therapy with or without chemotherapy for nasopharyngeal carcinoma: radiation therapy oncology group phase II trial 0225. J Clin Oncol 2009; 27: 3684-90.

22. Wolden SL, Chen WC, Pfister DG, Kraus DS, Berry SL, Zelefsky MJ. Intensitymodulated radiation therapy (IMRT) for nasopharyngeal cancer. Update of the Memorial Sloan-Kettering experience. Int J Radiat Oncol Biol Phys 2006; 64: 57-62. 